\title{
Uncalibrated 1D Projective Camera and 3D Affine Reconstruction of Lines
}

\author{
Long QUAN \\ CNRS-GRAVIR-INRIA \\ ZIRST - 655 avenue de l'Europe \\ 38330 Montbonnot, France \\ Email: Long.Quan@inrialpes.fr
}

\begin{abstract}
We describe a linear algorithm to recover $3 D$ affine shape/motion from line correspondences over three views with uncalibrated affine cameras. The key idea is the introduction of a one-dimensional projective camera. This converts the $3 D$ affine reconstruction of "lines" into $2 D$ projective reconstruction of "points". Using the full tensorial representation of three uncalibrated $1 D$ views, we prove that the $3 D$ affine reconstruction of lines from minimal data is unique up to a re-ordering of the views. $3 D$ affine line reconstruction can be performed by properly rescaling image coordinates instead of using projection matrices. The algorithm is validated on both simulated and real image sequences.
\end{abstract}

\section{Introduction}

Using line segments instead of points as features has attracted the attention of many researchers $[11,2,29,28$, $27,1]$ for various tasks such as pose estimation, stereo and structure from motion. In this paper, we are interested in structure from motion using line correspondences across mutiple images. A minimum of three views is essential for this, whereas two views suffice for point correspondences. In the case of calibrated perspective cameras, the main results on structure from line correspondences were established in $[11,22,2]$ : With at least six line correspondences over three views, nonlinear algorithms are possible. With at least thirteen lines over three views, a linear algorithm is possible. The basic idea of the thirteen-line linear algorithm is similar to that of the eight-point algorithm [12]: It is based on the introduction of a set of redundant intermediate parameters. This provides a very heavy overparametrization of the problem that definitely leads to the instability of the algorithm reported in [11]. The thirteenline algorithm was extended to uncalibrated camera case in $[7,27]$. The situation for uncalibrated camera case might be expected to be better, as more free parameters are needed. However, the 27 tensor components that are introduced as intermediate parameters are still subject to 9 complicated algebraic constraints. The algorithm can hardly be stable. A subsequent nonlinear optimization step is almost unavoidable to refine the solution $[2,11,22,7]$.

In parallel, there has been a lot of work [23, 26, 20, 16, $17,9,10,8,14,25]$ on structure from motion with simplified camera models varing from orthographic projections via weak and para-perspective to affine cameras, almost exclusively for point features. These simplified camera models provide a good approximation to perpsective projection when the depth of the object is small compared to the viewing distance. More importantly, they expose the ambiguities that arise when perspective effects diminish. In such cases, it is not only easier to use these simplified models but also advisable to do so, as by explicitly eliminating the ambiguities from the algorithm, one avoids computing parameters that are inherently ill-conditioned. Another important advantage of working with uncalibrated affine cameras is that the reconstruction is affine, rather than projective as with uncalibrated projective cameras.

Motivated on the one hand by the lack of satisfactory line-based algorithms for projective cameras and on the other by the fact that the affine camera is a good model for many practical cases, we investigated the properties of line projection by affine cameras and proposed a linear algorithm $[18,19]$ for affine structure from line correspondences.

This paper is an extension of our previous work in which the key advance introducing a one-dimensional projective camera was made. The previous work concentrated on the redundant data case to accomodate a factorization scheme for lines. We were unable to solve for the reconstruction ambiguity. In this paper, we use the same theoretical framework but concentrate on the minimal data case. Instead of using a projection matrix representation for reconstruction as in the previous work, we rely on a tensorial representation of multi-views with one-dimensional cameras. 
A complete analysis of the joint projection matrix reveals the important role of the "epipoles" which, although redundant with respect to the trilinear tensor, play a central role in disambiguating the reconstruction. This new developement allows us to finally prove that $3 \mathrm{D}$ affine reconstruction of lines with the minimal data is unique up to a re-ordering of views. Subsequently, a reconstruction algorithm based on the rescaling of image coordinates is proposed and validated on both simulated and real images.

Throughout the paper, tensors and matrices are denoted in upper case boldface, vectors in lower case boldface and scalars in either plain letters or lower case Greek.

\section{Review of the affine camera model for lines}

As far as perspective (pin-hole) cameras are concerned, the projection of a point $\mathbf{x}=(x, y, z, t)^{T}$ of $\mathcal{P}^{3}$ to a point $\mathbf{u}=(u, v, w)^{T}$ of $\mathcal{P}^{2}$ can be described by a $3 \times 4$ homogeneous projection matrix $\mathbf{P}$ :

$$
\lambda \mathbf{u}=\mathbf{P}_{3 \times 4} \mathbf{x} .
$$

For a restricted class of camera models, by setting the third row of the perspective camera $\mathbf{P}$ to $(0,0,0, \lambda)$, we obtain the affine camera initially introduced by Mundy and Zisserman in [15]

$$
\begin{aligned}
\mathbf{A}_{3 \times 4} & =\left(\begin{array}{cccc}
p_{11} & p_{12} & p_{13} & p_{14} \\
p_{21} & p_{22} & p_{23} & p_{24} \\
0 & 0 & 0 & p_{34}
\end{array}\right) \\
& \equiv\left(\begin{array}{cc}
\mathbf{M}_{2 \times 3} & \\
\mathbf{0}_{1 \times 3} & \mathbf{t}_{3 \times 1}
\end{array}\right) .
\end{aligned}
$$

This is the uncalibrated affine camera which emcompasses all the uncalibrated versions of the orthographic, weak perspective and paraperspective camera models.

Now consider a line in $\mathbb{R}^{3}$ through a point $\mathbf{x}_{0}$ with direction $\mathbf{d}_{x}$ :

$$
\mathbf{x}_{a}=\mathbf{x}_{0}+\lambda \mathbf{d}_{x} .
$$

The affine camera $\mathbf{A}_{3 \times 4}$ projects this to an image line:

$$
\mathbf{A}_{3 \times 4}\left(\begin{array}{c}
\mathbf{x} \\
1
\end{array}\right)=\left(\mathbf{M} \mathbf{x}_{0}+\mathbf{t}_{0}\right)+\lambda \mathbf{M d} \mathbf{d}_{x}=\mathbf{u}_{0}+\lambda \mathbf{M d} \mathbf{d}_{x}
$$

with direction

$$
\rho \mathbf{d}_{u}=\mathbf{M}_{2 \times 3} \mathbf{d}_{x},
$$

passing through the image point

$$
\mathbf{u}_{0} \equiv \mathbf{M x _ { 0 }}+\mathbf{t}_{0} .
$$

Equation (3) describes a linear mapping between directions of $3 \mathrm{D}$ lines and those of $2 \mathrm{D}$ lines. It can be derived even more directly using projective geometry, by considering that the line with direction $\mathbf{d}_{x}$ is the point at infinity $\mathbf{x}_{\infty}=\left(\mathbf{d}_{x}^{T}, 0\right)^{T}$ in $\mathcal{P}^{3}$ and the line with direction $\mathbf{d}_{u}$ is the point at infinity $\mathbf{u}_{\infty}$ in $\mathcal{P}^{2}$.

Comparing Equation (3) with Equation (1) which is a projection from $\mathcal{P}^{3}$ to $\mathcal{P}^{2}$, we see that Equation (3) is nothing but a projective projection from $\mathcal{P}^{2}$ to $\mathcal{P}^{1}$ if we consider the $3 \mathrm{D}$ and $2 \mathrm{D}$ directions of lines as $2 \mathrm{D}$ and $1 \mathrm{D}$ projective points. This means that the affine reconstruction of lines with a two-dimensional affine camera is equivalent to the projective reconstruction of points with a one-dimensional projective camera!

There have been many recent works $[3,5,24,13,4,6$, $21,22]$ on projective reconstruction and the geometry of multi-views of two dimensional uncalibrated cameras. Particularly, the tensorial formalism developed by Triggs [24] is very interesting and powerful. We are now extending this study to the case of the one-dimensional camera.

\section{Uncalibrated one-dimensional camera}

First, rewrite Equation (3) in the following form:

$$
\lambda \mathbf{u}=\mathbf{M}_{2 \times 3} \mathbf{x}
$$

in which we use $\mathbf{u}=\left(u_{1}, u_{2}\right)^{T}$ and $\mathbf{x}=\left(x_{1}, x_{2}, x_{3}\right)^{T}$ instead of $\mathbf{d}_{u}$ and $\mathbf{d}_{x}$ to stress that we are dealing with "points" in the projective spaces $\mathcal{P}^{2}$ and $\mathcal{P}^{1}$ rather than line directions in the vector spaces $\mathbb{R}^{3}$ and $\mathbb{R}^{2}$. This exactly describes a one-dimensional projective camera which projects a point $\mathbf{x}$ in $\mathcal{P}^{2}$ onto a point $\mathbf{u}$ in $\mathcal{P}^{1}$.

We now examine the matching constraints between multiple views of the same point. There is a constraint only for the case of 3 views.

Let the three views of the same point $\mathbf{x}$ be given as follows:

$$
\left\{\begin{aligned}
\lambda \mathbf{u} & =\mathbf{M} \mathbf{x}, \\
\lambda^{\prime} \mathbf{u}^{\prime} & =\mathbf{M}^{\prime} \mathbf{x}, \\
\lambda^{\prime \prime} \mathbf{u}^{\prime \prime} & =\mathbf{M}^{\prime \prime} \mathbf{x}
\end{aligned}\right.
$$

These can be rewritten in matrix form as

$$
\left(\begin{array}{cccc}
\mathbf{M} & \mathbf{u} & 0 & 0 \\
\mathbf{M}^{\prime} & 0 & \mathbf{u}^{\prime} & 0 \\
\mathbf{M}^{\prime \prime} & 0 & 0 & \mathbf{u}^{\prime \prime}
\end{array}\right)\left(\begin{array}{c}
\mathbf{x} \\
-\lambda \\
-\lambda^{\prime} \\
-\lambda^{\prime \prime}
\end{array}\right)=0
$$

which is the basic reconstruction equation for a onedimensional camera. The vector $\left(\mathbf{x},-\lambda,-\lambda^{\prime},-\lambda^{\prime \prime}\right)^{T}$ cannot be zero, and so

$$
\left|\begin{array}{cccc}
\mathbf{M} & \mathbf{u} & 0 & 0 \\
\mathbf{M}^{\prime} & 0 & \mathbf{u}^{\prime} & 0 \\
\mathbf{M}^{\prime \prime} & 0 & 0 & \mathbf{u}^{\prime \prime}
\end{array}\right|=0 .
$$


The expansion of this determinant produces a trilinear constraint of three views

$$
\sum_{i, j, k=1}^{2} T_{i j k} u_{i} u_{j}^{\prime} u_{k}^{\prime \prime}=0
$$

or in short

$$
\mathbf{T}_{2 \times 2 \times 2} \mathbf{u} \mathbf{u}^{\prime} \mathbf{u}^{\prime \prime}=0
$$

where $\mathbf{T}_{2 \times 2 \times 2}=\left(T_{i j k}\right)$ is a $2 \times 2 \times 2$ homogeneous tensor whose components $T_{i j k}$ are $3 \times 3$ minors of the following $6 \times 3$ joint projection matrix:

$$
\left(\begin{array}{c}
\mathbf{M} \\
\mathbf{M}^{\prime} \\
\mathbf{M}^{\prime \prime}
\end{array}\right)=\left(\begin{array}{c}
1 \\
2 \\
1^{\prime} \\
2^{\prime} \\
1^{\prime \prime} \\
2^{\prime \prime}
\end{array}\right)
$$

The components of the tensor can be made explicit as

$$
T_{i j k}=\left[\bar{i}^{\prime} \bar{k}^{\prime \prime}\right], \text { for } i, j^{\prime}, k^{\prime \prime}=1,2 \text {. }
$$

where the bracket $\left[i j^{\prime} k^{\prime \prime}\right]$ denotes the $3 \times 3$ minor of $i$-th, $j^{\prime}$ th and $k^{\prime \prime}$-th row vector of the above joint projection matrix and bar "-" in $\bar{i}, \bar{j}$ and $\bar{k}$ denotes the mapping

$$
(1,2) \mapsto(2,-1)
$$

It can be easily seen that any constraint obtained by adding further views reduces to a trilinearity. This proves the uniqueness of the trilinear constraint. Moreover, the $2 \times 2 \times 2$ homogeneous tensor $\mathbf{T}_{2 \times 2 \times 2}$ has $7=2 \times 2 \times 2-1$ d.o.f., so it is a minimal parametrization of three views since three views have exactly

$$
3 \times(2 \times 3-1)-(3 \times 3-1)=7
$$

d.o.f., up to a projective transformation in $\mathcal{P}^{2}$.

Each correspondence over three views gives one linear constraint on the tensor components $T_{i j k}$. With at least 7 points in $\mathcal{P}^{1}$, the tensor components $T_{i j k}$ can be estimated linearly.

At this point, we have obtained a remarkable result that for the one-dimensional projective camera, the trilinear tensor encapsulates exactly the information needed for projective reconstruction in $\mathcal{P}^{2}$. Namely, it is the unique matching constraint, it minimally parametrizes the three views and it can be estimated linearly. Contrast this to the 2D image case in which the multilinear constraints are algebraically redundant and the linear estimation is only an approximation based on over-parametrization.

\subsection{D projective reconstruction by rescaling}

According to Triggs [24], the projective reconstruction in $\mathcal{P}^{3}$ can be viewed as being equivalent to the rescaling of the image points in $\mathcal{P}^{2}$. We have just proven that recovering the directions of affine lines in $3 \mathrm{D}$ space is equivalent to $2 \mathrm{D}$ projective reconstruction from one-dimensional projective images. Therefore, a reconstruction of the directions of 3D affine lines can be obtained by rescaling the direction vectors of image lines, viewed as points of $\mathcal{P}^{1}$.

For each 1D image point through in views ( $c f$. Equation (5)), the scale factors $\lambda, \lambda^{\prime}$ and $\lambda^{\prime \prime}$-taken individuallyare arbitrary: However, taken as a whole $\left(\lambda \mathbf{u}, \lambda^{\prime} \mathbf{u}^{\prime}, \lambda^{\prime \prime} \mathbf{u}^{\prime \prime}\right)^{T}$, they encode the projective structure of the points $\mathbf{x}$ in $\mathcal{P}^{2}$. One way to explicitly recover the scale factors $\left(\lambda, \lambda^{\prime}, \lambda^{\prime \prime}\right)^{T}$ is to notice that the rescaled image coordinates $\left(\lambda \mathbf{u}, \lambda^{\prime} \mathbf{u}^{\prime}, \lambda^{\prime \prime} \mathbf{u}^{\prime \prime}\right)^{T}$ should lie in the joint image, or alternatively to observe the following matrix identity:

$$
\left(\begin{array}{cc}
\mathbf{M} & \lambda \mathbf{u} \\
\mathbf{M}^{\prime} & \lambda^{\prime} \mathbf{u}^{\prime} \\
\mathbf{M}^{\prime \prime} & \lambda^{\prime \prime} \mathbf{u}^{\prime \prime}
\end{array}\right)=\left(\begin{array}{c}
\mathbf{M} \\
\mathbf{M}^{\prime} \\
\mathbf{M}^{\prime \prime}
\end{array}\right)\left(\begin{array}{ll}
\mathbf{I}_{3 \times 3} & \mathbf{x}
\end{array}\right) .
$$

The rank of the left matrix is therefore at most 3. All $4 \times 4$ minors vanish. Expanding by cofactors in the last column gives homogeneous linear equations in the components of $\lambda \mathbf{u}, \lambda^{\prime} \mathbf{u}^{\prime}$ and $\lambda^{\prime \prime} \mathbf{u}^{\prime \prime}$ with coefficients that are $3 \times 3$ minors of the joint projection matrix:

$$
\mathbf{T}_{\cdot j k}(\lambda \mathbf{u})-\mathbf{e}_{1}^{\prime \prime}\left(\lambda^{\prime} \mathbf{u}^{\prime}\right)^{T}+\mathbf{e}_{1}^{\prime}\left(\lambda^{\prime \prime} \mathbf{u}^{\prime \prime}\right)^{T}=\mathbf{0}_{2 \times 2},
$$

where $\mathbf{T}_{{ }_{j k}} \mathbf{u}$ is for $\sum_{i=1}^{2} \mathbf{T}_{i j k} \mathbf{u}^{i}$, a $2 \times 2$ matrix.

There are two types of minors: Those involving three views with one row from each view and those involving two views with two rows from one view and one from the other. The first type gives the 8 components of the tensor $\mathbf{T}_{2 \times 2 \times 2}$ and the second type gives 12 components of the "epipoles" $\mathbf{e}_{1}, \mathbf{e}_{2}, \mathbf{e}_{1}^{\prime}, \mathbf{e}_{2}^{\prime}, \mathbf{e}_{1}^{\prime \prime}, \mathbf{e}_{2}^{\prime \prime}$. The epipoles are defined by analogy with the $2 \mathrm{D}$ camera case, as the projection of one projection center onto another view.

At present we only know $T_{i j k}$-the epipoles are still unknown. To find the rescaling factors for projective reconstrucion, we need to solve for the epipoles. One way to proceed is as follows. Taking $\mathbf{x}$ to be the projection center of the second view $\mathbf{o}^{\prime}$, and projecting into the three views, Equation (10) reduces to

$$
\lambda \mathbf{T}_{\cdot j k} \mathbf{e}_{2}=-\lambda^{\prime \prime} \mathbf{e}_{1}^{\prime} \mathbf{e}_{2}^{\prime \prime T}
$$

As $\mathbf{e}_{1}^{\prime} \mathbf{e}^{\prime \prime T}$ has rank 1 , so does $\mathbf{T} \cdot{ }_{j k} \mathbf{e}_{2}$. Its $2 \times 2$ determinant must vanish, i.e.

$$
\left|\mathbf{T}_{\cdot j k} \mathbf{e}_{2}\right|=0
$$


As each entry of the $2 \times 2$ matrix is homogeneous linear in $\mathbf{e}_{2}=\left(u_{1}, u_{2}\right)^{T}$, the expansion of $\left|\mathbf{T}_{\cdot j k} \mathbf{e}_{2}\right|$ gives a homogeneous quadratic

$$
\alpha u_{1}^{2}+\beta u_{1} u_{2}+\gamma u_{2}^{2}=0,
$$

where $\alpha, \beta, \gamma$ are known in terms of $T_{i j k}$.

Doing the same thing with the projection center of the third view $\mathbf{o}^{\prime \prime}$ gives

$$
\lambda \mathbf{T}_{\cdot j k} \mathbf{e}_{3}=\lambda^{\prime} \mathbf{e}_{1}^{\prime \prime} \mathbf{e}_{3}^{\prime T} .
$$

and hence

$$
\left|\mathbf{T}_{\cdot j k} \mathbf{e}_{3}\right|=0 \text {. }
$$

In other words, it leads to exactly the same quadratic equation (11) with $\mathbf{e}_{3}$ replacing $\mathbf{e}_{2}$. The two solutions of the quadratic (11) are $\mathbf{e}_{2}$ and $\mathbf{e}_{3}$-only the ordering remains ambiguous.

The other epipoles are easily obtained, $\mathbf{e}_{1}^{\prime}$ and $\mathbf{e}_{2}^{\prime \prime}$ by factorizing the matrix $\mathbf{T}_{\cdot j k} \mathbf{e}_{2}$ and $\mathbf{e}_{1}^{\prime \prime}$ and $\mathbf{e}_{1}^{\prime}$ by factorizing $\mathbf{T} \cdot{ }_{j k} \mathbf{e}_{3}$.

If the first solution set is

$$
\left\{\tilde{\mathbf{e}}_{2}, \tilde{\mathbf{e}}_{1}^{\prime}, \tilde{\mathbf{e}}_{2}^{\prime \prime}, \tilde{\mathbf{e}}_{3}, \tilde{\mathbf{e}}_{1}^{\prime \prime}, \tilde{\mathbf{e}}_{3}^{\prime}\right\},
$$

the reordering gives the second solution set

$$
\left\{\mathbf{e}_{3}=\tilde{\mathbf{e}}_{2}, \mathbf{e}_{1}^{\prime \prime}=\tilde{\mathbf{e}}_{1}^{\prime}, \mathbf{e}_{3}^{\prime}=\tilde{\mathbf{e}}_{2}^{\prime \prime}, \mathbf{e}_{2}=\tilde{\mathbf{e}}_{3}^{\prime \prime}, \mathbf{e}_{1}^{\prime}=\tilde{\mathbf{e}}_{1}^{\prime \prime}, \mathbf{e}_{2}^{\prime \prime}=\tilde{\mathbf{e}}_{3}^{\prime}\right\}
$$

Once all the epipoles have been recovered, the scale factors of the image "points" for 3D direction reconstruction can easily be recovered by solving the linear homogeneous equation (10).

\subsection{Retrieving normal forms for projection matri- ces}

The geometry of the three views is most conveniently, and completely represented by the projection matrices associated with each view. In the previous section, the trilinear tensor was expressed in terms of the projection matrices. Now we seek a map from the trilinear tensor representation back to the projection matrix representation of the three views.

Without loss of generality, we can always take the following normal forms for the 3 projection matrices

$$
\begin{aligned}
\mathbf{M} & =\left(\begin{array}{ll}
\mathbf{I}_{2 \times 2} & \mathbf{0}
\end{array}\right), \\
\mathbf{M}^{\prime} & =\left(\begin{array}{ll}
\mathbf{A}_{2 \times 2} & \mathbf{c}
\end{array}\right), \\
\mathbf{M}^{\prime \prime} & =\left(\begin{array}{ll}
\mathbf{D}_{2 \times 2} & \mathbf{f}
\end{array}\right) .
\end{aligned}
$$

It is straightforward to verify that the projection center of the first view is $\operatorname{Ker}\left(\mathbf{M}_{1}\right)=(0,0,1)^{T}$, so that $\mathbf{e}_{1}^{\prime}=\mathbf{c}$ and $\mathbf{e}_{1}^{\prime \prime}=\mathbf{f}$.

Now, the trilinear tensor $\left(T_{i j k}\right)$ can be exhibited as

$$
\lambda T_{i j k}=(-1)^{i+1}\left(d_{\bar{k} i} c_{\bar{j}}-a_{\bar{j} i} f_{\bar{k}}\right) .
$$

As $\mathbf{c}$ and $\mathbf{f}$ are known, $a_{i j}$ and $d_{i j}$ can be solved linearly from the eight homogeneous equations of (13).

Note that in our previous work [18], we recovered the projection matrices nonlinearly without knowing epipoles, whereas here we recover them linearly using the epipoles.

\section{Uncalibrated translations and affine shape}

To recover the full affine structure of the lines, we still need to find the vector $\mathbf{t}_{3 \times 1}$ of the affine cameras defined in (2). These represent the image translation and magnification components of the camera. Recall that line correspondences from two views do not impose any constraints on camera motion: The minimum number of views required is three. The recovery of the uncalibrated translations is essentially linear once the uncalibrated rotations have been recovered. A detailed linear algorithm is developed in our previous work $[18,19]$.

The final reconstruction step of lines can be easily formulated as a subspace selection and solved by SVD $[18,19]$.

\section{Affine-structure-from-lines theorem}

In view of the results obtained above, we can establish the following.

For the recovery of affine shape and affine motion from line correspondences with an uncalibrated affine camera, the minimum number of views needed is three and the minimum number of lines required is seven for a linear solution. The recovery is unique up to a re-ordering of the views.

This result can be compared with that of Koenderink and Van Doorn [9] for affine structure with a minimum of two views and five points.

\section{Experimental results}

The algorithm presented in this paper has been validated with both simulated and real image sequences. Due to lack of space, only an experiment based on real images will be presented.

A Fujinon/Photometrics CCD camera is used to aquire a sequence of images of a box of size $12 \times 12 \times 12.65 \mathrm{~cm}$. The image resolution is $576 \times 384$. A Canny-like edge detector is first applied to each image. The contour points are then linked and fitted to line segments by least squares. Line correspondences across three views are selected by hand. A total of 46 lines is selected, as shown in Figure 1.

The reconstruction algorithm generates infinite 3D lines. To find 3D line segments, we reproject the 3D lines into one 


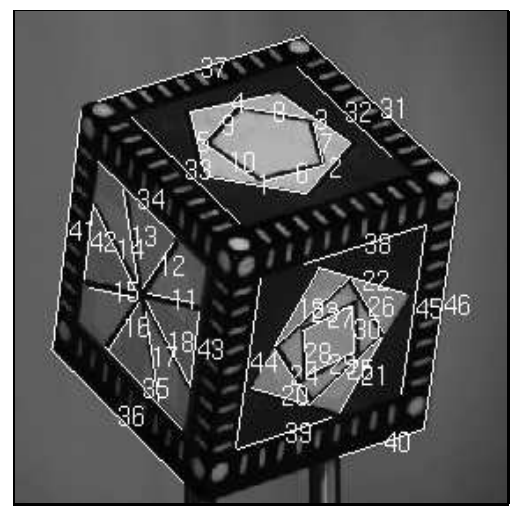

Figure 1. One image of the sequence and the extracted line segments.

image plane, then project the corresponding original image line segment orthogonally onto the reprojected line and finally back-project the resulting reprojected line segment to 3D space.

Figure 2 shows two views of the reconstructed 3D line segments. We note that the affine structure of the box is almost perfectly recovered. An average residual error of one tenth of a pixel is achieved.

The affine structures obtained can be converted to Euclidean ones (up to a global scaling factor) as soon as we know the aspect ratio of the camera [17]. Figure 3 shows the rectified affine shape. The two sides of the box are accurately orthogonal to each other.

\section{Discussion}

A linear structure from motion algorithm for lines with uncalibrated affine cameras has been presented, based on an analysis of the geometry of uncalibrated multiple views in $1 \mathrm{D}$ cameras. The algorithm requires a minimum number of seven line correspondences over three views. It has also been proven that the affine reconstruction is unique up to a re-ordering of views with the minimal data. The linear algorithm is not based on the over-parametrization used for perspective cameras. This gives the intrinsic stability of the algorithm. The previous results of Koenderink and Van Doorn [9] on affine structure from motion using point correspondences are therefore extended to line correspondences. Experimental results based on real and simulated image sequences demonstrate the accuracy and the stability of the method.

As the algorithm presented in this paper is developed within the framework suggested in [17] for points, it is straightforward to integrate both points and lines into the same approach.
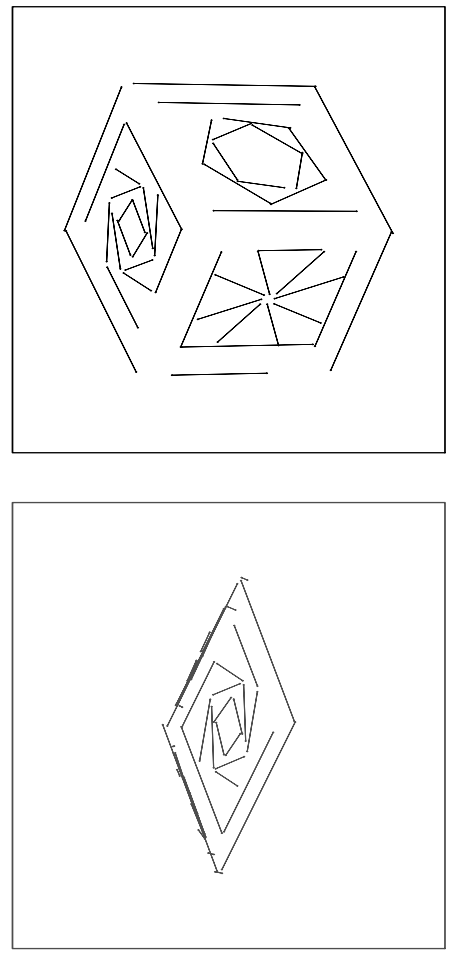

Figure 2. Reconstructed line segments: a general view and a top view

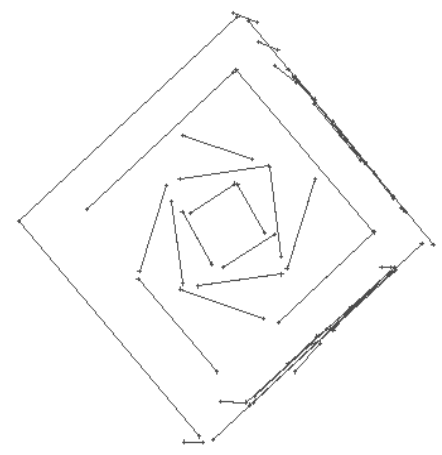

Figure 3. A side view rectified using the known aspect ratio of the camera. 


\section{Acknowledgement}

This work was partly supported by European project CUMULI which is gratefully acknowledged. The key idea of using the full tensorial approach to study the line reconstruction ambiguity comes from a discussion with B. Triggs who also provided much aid on tensor representation and interesting discussion on his work. We would also like to thank Roger Mohr and Peter Sturm for many fruitful discussions. The image sequences were taken in CMU with the help of D. Morris and N. Chiba.

\section{References}

[1] D. Dementhon and L.S. Davis. Model-based object pose in 25 lines of code. IJCV , 15(1/2):123-141, 1995.

[2] O.D. Faugeras, F. Lustman, and G. Toscani. Motion and structure from point and line matches. In ICCV, June 1987.

[3] O. Faugeras. What can be seen in three dimensions with an uncalibrated stereo rig? In ECCV, pages 563-578. May 1992.

[4] O. Faugeras and B. Mourrain. About the correspondence of points between $\mathrm{N}$ images. In Workshop on Representations of Visual Scenes, June 1995.

[5] R. Hartley, R. Gupta, and T. Chang. Stereo from uncalibrated cameras. In CVPR, pages 761-764, 1992.

[6] R. Hartley. Lines and Points in Three Views - An Integrated Approach. Technical report, G.E. CRD, 1994.

[7] R.I. Hartley. Projective reconstruction from line correspondences. In $C V P R, 1994$.

[8] R. Horaud, S. Christy, and F. Dornaika. Object Pose: the Link between Weak Perspective, Para Perspective, and Full Perspective. Technical report, Inria, September 1994.

[9] J.J. Koenderink and A. J. Van Doorn. Affine structure from motion. Technical report, Utrecht University, Utrecht, The Netherlands, October 1989.

[10] C.H. Lee and T. Huang. Finding point correspondences and determining motion of a rigid object from two weak perspective views. CVGIP, 52:309-327, 1990.

[11] Y. Liu and T.S. Huang. A linear algorithm for motion estimation using straight line correspondences. CVGIP, 44(1):3557, October 1988.

[12] H.C. Longuet-Higgins, A computer program for reconstructing a scene from two projections. in Nature, vol. 293, pp. 133-135. XX, September 1981.

[13] Q.T. Luong and T. Vieville. Canonic Representations for the Geometries of Multiple Projective Views. In ECCV, pages 589-599. May 1994.

[14] Ph.F. McLauchlan, I.D. Reid and D.W. Murray. Recursive affine structure and motion from image sequences. In ECCV, pages 217-224. May 1994.

[15] J.L. Mundy and A. Zisserman, editors. Geometric Invariance in Computer Vision. MIT Press, Cambridge, Massachusetts, USA, 1992.
[16] C. J. Poelman and T. Kanade. A paraperspective factorization method for shape and motion recovery. In $E C C V$, pages 97-108, May 1994.

[17] L. Quan. Self-calibration of an affine camera from multiple views. IJCV, 1996.

[18] L. Quan and T. Kanade. A factorization method for shape and motion from line correspondences. In CVPR, June, 1996.

[19] L. Quan and T. Kanade. Affine Structure from Line Correspondences with Uncalibrated Af fine Cameras. To appear in IEEE T-PAMI, 1997.

[20] L.S. Shapiro, A. Zisserman, and M. Brady. Motion from point matches using affine epipolar geometry. IJCV, 1994.

[21] A. Shashua. Algebraic functions for recognition. IEEE $T$ PAMI, 1994. in press.

[22] M. Spetsakis and J. Aloimonos. A Unified theory of structure from motion. In Proceedings DARPA IU Workshop, 1990.

[23] C. Tomasi and T. Kanade. Shape and motion from image streams under orthography: A factorization method. IJCV, 9(2):137-154, 1992.

[24] B. Triggs. The geometry of projective reconstruction I: Matching constraints and the joint image. In ICCV, 1995.

[25] S. Ullman and R. Basri. Recognition by linear combinations of models. IEEE T-PAMI, 13(10):992-1006, 1991.

[26] D. Weinshall and C. Tomasi. Linear and incremental acquisition of invariant shape models from image sequences. In ICCV. 1993.

[27] T. Vieville and O. Faugeras and Q.T. Luong. Motion of points and lines in the uncalibrated case. IJCV, 7(3):211241, 1996.

[28] J. Weng and T.S. Huang and N. Ahuja. Motion and structure from line correspondences: Closed-form solution, uniqueness, and optimization. IEEE T-PAMI, 14(3):318-336, 1992.

[29] Z. Zhang and O. Faugeras. Three-dimensional motion computation and object segmentation in a long sequence of stereo frames. IJCV, 7(3):211-241, 1992. 